\title{
Elemental, Nano-Sized (100-500 nm) Selenium Production by Probiotic Lactic Acid Bacteria
}

\author{
Péter Eszenyi, Attila Sztrik, Beáta Babka, and József Prokisch
}

\begin{abstract}
Selenium is well known as an essential trace element since the 20th century, but it can be overdosed easily because of its toxicity. According to the present regulations [1] only the potentially most harmful, inorganic selenium salts can be added to any comestibles. Elemental selenium is considered as the least toxical of all selenium forms and in the same time supplementation with its nano-size particles has the same or better bioavailability compared to its salts. In our experiments we managed to produce nano-size $(100-500 \mathrm{~nm})$ elemental selenium by using probiotic yogurt bacteria in a fermentation procedure. We developed the laboratory technology of product purification and recovery from bacteria.
\end{abstract}

Index Terms-Antioxidant, Food Supplement, Lactic Acid Bacteria, Nanosphere, Selenium.

\section{INTRODUCTION}

\section{A. Selenium forms in Our Environment}

In the geosphere there is not enough natural elemental selenium that worth to exploit with economic benefit. Usually we can find relatively large amount of elemental selenium in ores, few carbon layers of the soil and in the soils of volcanic areas. The most common selenium forms of our biosphere are the inorganic selenium salts. These salts leach easily from the soil and become available for the plants by the groundwater [2]. In alkaline environment a termodinamically stable form of selenium is the selenate ion $\left(\mathrm{SeO}_{4}{ }^{2}-\right)$. Selenates have better soluble but worse absorption properties than selenites. Selenite $\left(\mathrm{SeO}_{3}{ }^{2}-\right)$ occurs in neutral $\mathrm{pH}$ environment and it is less soluble compared to selenate. Selenite also has the ability to absorb to particles dynamically and can be reduced to elemental selenium $\left(\mathrm{Se}^{0}\right)$ by chemical or biological ways. Selenides $\left(\mathrm{Se}^{2-}\right)$ and the selenium enriched sulphides occur in reductive or acidic environment, have weak soluble and oxidating properties, plants and animals hardly can take them up.

\section{B. Selenium forms in the Biological Systems}

Se proteins, synthesized by plants and animals contain $\mathrm{Se}-\mathrm{Met}$; which is the principal form of $\mathrm{Se}$ in cereals and other plants. Animals do not distinguish between Se-Met and its S-analogue Met. Both amino acids are incorporated in proteins via the same enzymatic pathway [3] In plants selenate is much more easily transported than selenite or organic Se [4]. Se distribution over several plant compartments depends on plant species, developmental phase, physiological condition, form, and concentration of the $\mathrm{Se}$ available, and the presence of other substances,

Manuscript received June 13, 2011; revised June 29, 2011.

The authors are with Institute of Bio and Environmental Energetics University of Debrecen, Centre for Agricultural Sciences and Engineering H-4032 Debrecen, Böszörményi út 138.

Hungary eszenyipeter@agr.unideb.hu especially sulfates. Selenoproteins are common in Brazil nuts and Se-accumulating plants, like onion, garlic, species of brassica genus and mushrooms. Certain yeasts, like Saccharomyces cerevisiae, have the ability to accumulate and transform high concentration of selenium, when we add $\mathrm{Na}_{2} \mathrm{SeO}_{3}$ to the growth medium. Similarly to plants, $\mathrm{Se}$ is mainly incorporated as Se-Met in their proteins. [5]. Due to its low cost and its ability to synthetize selenoproteins yeasts can be well utilized for nutritional supplementation.

\section{Biological Production of Nano Particles}

Researchers just started to recognise in the recent years the importance of the ability of certain microorganisms to produce nano-sized particles in the course of their metabolism. Many elements in trace concentrations are essential for the growth and reproduction of plants, animals and microorganisms, however these elements easily become toxic at concentrations higher than the physiological level. Scientists have shown that many plants and bacteria can actively uptake and reduce metal ions from soil and solutions. Nair and Pradeep [6] used Lactic acid bacteria in buttermilk whey to produce gold-silver composite materials. These alloy materials, in submicron dimensions, form several well-defined crystal morphologies, and this crystal growth does not affect the viability of the bacteria. Magneto-tactic bacteria, for instance, have intracellular magnetic structures, the magnetosomes, which comprise nanometer-sized, membrane-bound crystals of the magnetic iron minerals magnetite $\left(\mathrm{Fe}_{3} \mathrm{O}_{4}\right)$ or greigite $\left(\mathrm{Fe}_{3} \mathrm{~S}_{4}\right)$. Magnetosomes [7] are the results of a mineralization process with biological control over the accumulation of iron and the deposition of the mineral particle with specific size and orientation within a membrane vesicle at specific locations in the cell. Moon [8]. discovered that certain thermophyl and psychrotolerant metal-reducer bacteria (Shewanella sp.; Thermoanaerobacter $s p$ ) are able to produce copious amounts of extra-cellular metal (M)-substituted magnetite nano-particles using akaganeite and dopants of dissolved form.

Not only the bacteria but also the fungi are able to synthetize nano-sized products. Fungi, due to their metal accumulation and tolerant ability, were placed into the centre of attention of nano-particle production researches [9]. Their economic viability, ease in scale up in solid substrate fermentations and large-scale secretion of extracellular enzymes, makes them advantageous for nano-particle production [10].

As a new field of nanobiotechnology, besides bacteria and fungi, plants are also able to produce nano-particles. In a study scientists [10] describe that they managed to fabric gold nano-particles using live plants. In their experiment they grew Alfalfa plants in an $\mathrm{AuCl}_{4}$ rich environment, plants absorbed the $\mathrm{Au}$ metal successfully, that was 
confirmed by X-ray absorption studies (XAS), and transmission electron microscopy (TEM). These gold clusters surrounded by a shell of organic ligands covalently attach to proteins or other biological substances. Atomic resolution analysis confirmed that $\mathrm{Au}$ nanoparticles inside the plant are in a crystalline state and pure gold.

\section{Nano-Sized Elemental Selenium}

As we mentioned above elemental selenium rarely occurs in our environment, mainly in the soils with anaerob conditions, in stable form, and doesn't or hardly dissolves in water. Thanks to these properties elemental selenium is less toxic compared to other selenium forms. First it was generally considered about elemental selenium to be biologically inert, but researchers proved that Nano-Se has similar bioavailability to other selenium forms. Zhang [11] found large difference in acute toxicity between Nano-se and selenite. In their study they compared the short-term toxicity of the two Se forms in mice by orally administering high doses of Se. Their results showed that Nano-Se was less toxic than selenite in terms of suppressing growth, liver toxicity, and antioxidant status, but has similar bioavailability. Nano-size, in the range of 100-500nm, also helps the better absorption of selenium into plants, animals, humans and microorganisms. Nano-sized selenium production has two ways: chemical and biological.

In the chemical way scientists don't use any living/ organic material, they usually start from inorganic selenite and add some reducer agent, like ascorbic acid. Or there is a wet chemical method [12] for the preparation of $\alpha$ monoclinic selenium nanowires. The method is the reduction of sodium selenite with glutathione $(\mathrm{GSH})$ at room temperature in aqueous solution. Glutathione, having a thiol group, reacts with sodium selenite to form selenodiglutathione (GSSeSG), which decomposes to produce selenium molecules and diglutahtione (GSSG). Selenium molecules aggregate together to form selenium nanospheres. Positive effects of chemically synthesized selenium nanospheres were examined in several experiments succesfully. Wang [13] was led to the conclusion in 2007 based on animal tests, nano selenium (Nano-Se) is a very effective antioxidant, without high toxicity properties which is typical for other selenium forms. Nano selenium has at least the same effect on activating glutathione peroxidase and thioredoxin reductase enzymes as selenoproteines has, but acording to the $\mathrm{LD}_{50}$ this form is less toxical, it doesn't trigger acute liver injury and short term toxications. Furthermore nano selenium accumulates less in the treated mice and activates glutathione-Stransferase more effectively than selenoproteins, independently from the level of toxication.

One of the biological ways is presented by Oremland [14] who reported about the production of elemental selenium nano-spheres, that our laboratory also focuses on, in case of specific bacteria. In 2004 scientists described that a selenium reducer anaerob bacteria, that inspires toxical seleium oxianions is able to produce and accumulate extracellular elemental selenium $[\mathrm{Se}(0)]$. In their experiments they later used three, phisiologically and phylogenetically diverse bacterium species in order to look into the phenomenon. Sulfurospirillum barnesii, Bacillus selenitireducens and Selenihalanaerobacter shriftii. During the cell growth they were using selenium oxianions as electron acceptors, following this each microorganisms produced extracellular, uniform sized and shaped approx. 300nm diameter selenium spheres with monoclinic crystalline structure. Not only extracellular but also intracellular elemental selenium production was observed. Fesharaki [15] (2010) in his study describe that they developed the biological synthesis and recovery on selenium nano-particles from bacterial cells. They tested a strain of Klebsiella pneumoniae to produce elemental selenium from selenium chloride. These particles in the range of 100$550 \mathrm{~nm}$ proved to be very resistant against high temperature and pressure, because there were no chemical changes during sterilization at $121^{\circ} \mathrm{C}$ and $17 \mathrm{psi}$ for 20 minutes. Therefore wet heat sterilization can be used for the recovery of elemental selenium from bacterial cells. Optical properties of elemental selenium produced this way were very distinct compared to the chemically synthesized selenium, we can conclude that elemental selenium production in microbial way results more unique, standard structured nanospheres.

\section{E. Our Scientific Concept and Goals}

Studying behaviour of selenium during yoghurt producing process led researchers in our laboratory to the discovery that certain Lactic acid bacteria species are able to reduce the selenite in toxical concentration into nano-sized, elemental selenium spheres. This is the first technology in which lactic acid and other probiotic bacteria produce elemental selenium in laboratory environment. (patent: [16]). We tested Lactobacillus sp., Bifidobacter sp., Streptococcus thermofilus and different kind of mixtures from various strains. During the electronmicroscopic analysis it became clear that each bacteria strain produce selenium spheres in different size ranges as follows: Lactobacillus sp. :100200nm, Bifidobacter sp. 400-500nm, Streptococcus thermofilus: $50-100 \mathrm{~nm}$. By the selection of bacteria used for production of high purity elemental red and grey selenium it was a very important factor to choose bacteria being already used and allowed to use in the food industry because our new product in this manner meets the strict quality requirements regarding for food supplements, additives. This technology seems to be more effective than the chemical synthesis, because it results relatively regular and uniform sized, high purity selenium spheres (100-500 nm, bacterium depending), production process is cheaper, faster and parameters can be controlled better. One of our future plans is to share this novel biological Nano-Se as food supplements, forage additives or plant nutritions.

Summarizing the goals, our fundamental endeavor was to produce a non-toxical selenium form by the means of granted microbes, which are used in the food industry, without any non-desired side effects, enhancing resistivity toward illnesses, increasing the blood antioxidant level and boosting the quality and quantity of agricultural products as a fertilizer or forage supplement.

According to the utilization we can differentiate two, nano-selenium containing products. First is the purified elemental nano-selenium (Nano-Se), that is mainly used for further laboratory and nanotechnological experiments, but 
later we intend to produce it for utilizing in different kind of medicinal supplements or fertilizer additives in industrial scale. The second product is the lacto selenium (LactoMicroSel) which is produced by the lactic acid bacteria in the yoghurt making process. Lactselenium is mainly used for forage additive experiments in case of mice and sheeps, in the future this form could be used in form of yoghurt powder as a forage additive. But another possible way of utilization for human selenium supplementation could be to eat a cup of delicious lactoselenium enriched yoghurt

\section{MATERIALS AND MethodS}

\section{A. Strains}

The following bacteria strains (from Chr. Hansen) were used in our experiments: Lactobacillus casei; Streptococcus thermophilus; Bifidobacterium BB-12; Lactobacillus acidophilus (LA-5); Lactobacillus helveticus (LH-B02). Only fresh cultures were used after three times passing.

\section{B. Culture Media}

For the adequate growth of the bacteria we need an appropriate culture medium, that contains carbon source, nitrogen source, inorganic ions and, if necessary, other organic materials. The applied organic micronutrient source advantageously contains sufficient quantity of desired materials, such as vitamin B1 or L-isoleucine, or, alternatively, yeast extract $\max$ be used. In addition, potassium phosphate, magnesium sulphate, ferrous ions and manganous ions can be added. The medium may also contain buffer and antifoaming agent. The commercially available media the MRS Broth or M17 are suitable for the Lactic acid bacteria or the $S$. thermophilus respectively. We used MRS in case of purified Nano-Se.

In case of LactoMicroSel production we use fresh cow milk as culture medium instead of MRS. Before the innoculation milk must be skimmed with a industrial milk centrifuge in order to reduce milk fat content under $0,5 \%$, which is appropriate for further steps of the process.

\section{Nano-Selenium production}

1) Production and Recovery of Purified Nano-Selenium

We dissolve the MRS, 5,5grams in $100 \mathrm{ml}$ purified water, then boil the medium for 30 minutes $120^{\circ} \mathrm{C}$. After cooling down to $25^{\circ} \mathrm{C}$ we add the selenium source and the inoculum. As selenium source we use sodium hydrogen selenite $\left(\mathrm{NaHSeO}_{3}\right)$ in a $10.000 . \mathrm{mg} / \mathrm{l}$ stock solution. We add $20 \mathrm{ml}$ of this stock solution to $980 \mathrm{ml}$ of MRS solution, it means around $200 \mathrm{mg} / \mathrm{l}$ concentration. From the fresh bacterium culture (previously stored at $4{ }^{\circ} \mathrm{C}$ for maximum 3-4 weeks) we add $10 \mathrm{ml}$ to $1000 \mathrm{ml}$ of selenite containing MRS solution. For the inoculation we choose one or the mixture of the following strains: Lactobacillus acidophillus, Streptococcus thermophilus and Lactobacillus casei. It is not very important to adjust the $\mathrm{pH}$ of the culture medium, but we usually check it, it is around 7-8 in the beginning and decreases to around 3-4 to the end of the fermentation process. After we added all components to the medium, we place the fermentation bottle into the shaking incubator for $36-48$ hours at $37{ }^{\circ} \mathrm{C}$ (optimum temperature for lactic acid bacteria full reproduction cycle). To the end of the fermentation process the culture medium become red, because of the produced elemental selenium. Next step is to centrifuge the medium at $6.000 \mathrm{rpm}$ for $10-15$ minutes and then discard the supernatant and we uptake the pellets in purified water. The formation mechanism of elemental selenium is mainly intracellular in lactic acid bacteria (1/A. picture), that is why we have to digest their very resistant cell wall. The most effective and money saving (compared to enzymatic cell wall digestion) method is to use high concentration of hydrochloric acid $(37 \% \mathrm{HCl})$. We add around $1,5 \mathrm{x}$ acid to the nanoselenium sample (e.g.: $150 \mathrm{ml}$ acid to $100 \mathrm{ml}$ sample). This acidic hydrolysis takes approx. five days at room temperature. After this step we have to get rid of the acid, accordingly we centrifuge $(6.000 \mathrm{rpm}$ for 10 15 minutes) the sample more times and wash with purified water until its $\mathrm{pH}$ returns to neutral. We put the samples into the ultrasonic for 10-15 minutes in order to disintegrate the cohesive selenium spheres. As a last step we use vacuum filter to get rid of the rest of the bacteria cell wall. We use one plastic filter layer and two paper layers. In the end we check the quality of the samples by visual or laser controlling whether they meet the requirements. Good quality samples have typical, nano-particle like light diffraction properties. For further utilization samples (1/B. picture) are stored at $4{ }^{\circ} \mathrm{C}$.

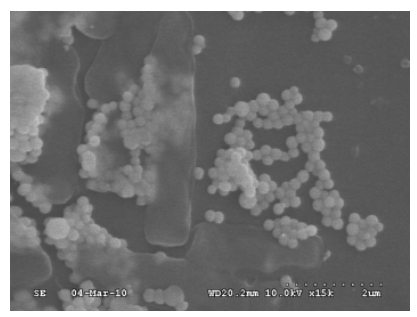

1/A picture. selenium spheres releasing from bacteria

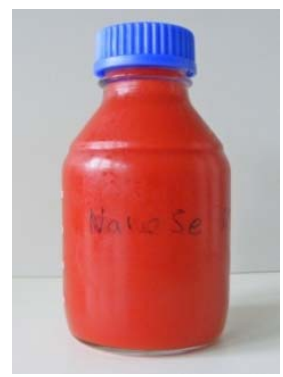

1/B picture. purified selenium suspension

\section{2) Production of Lactomicrosel (Selenium Enriched} Yoghurt Powder)

The selenium source is the sodium hydrogen selenite $\left(\mathrm{NaHSeO}_{3}\right)$ as well, but in powder form. For the inoculation we also choose one or the mixture of the following strains: Lactobacillus acidophillus, Streptococcus thermophilus and Lactobacillus casei. After we added all components to the medium, we place the cans or buckets into the shaking incubator for $36-48$ hours at $37{ }^{\circ} \mathrm{C}$. In the end of the fermentation process we have dense, selenium rich pink or red yoghurt. We put the yoghurt into the centrifuge at 6.000 rpm for 10-15 minutes in order to get rid of most of the water. We put this solid phase (2/A. picture) into the dryer (or into the freeze dryer, but this is more expensive) and let it dry for $12-16$ hours at $50-60^{\circ} \mathrm{C}$. After grinding and 
packing the lactoselenium (2/B. picture) into plastic boxes it is ready for the experiments, to mix it into the forage. Yield of the production process is: from 10liters of milk we get 300-400 grams of lactomicrosel with a concentration rate of $1500-3500 \mathrm{mg} / \mathrm{kg}$. As a result of our measures selenium in the lactoselenium is manly $(>95 \%)$ in a form of nanoselenium and the rest $(<5 \%)$ is organic selenium.

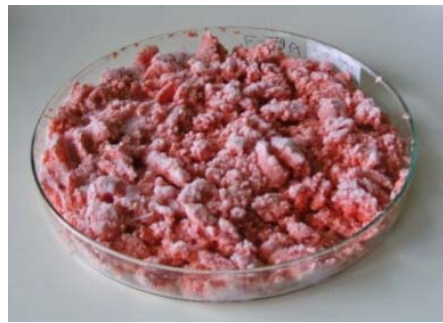

2/A picture. centrifuged selenium rich yoghurt, before drying

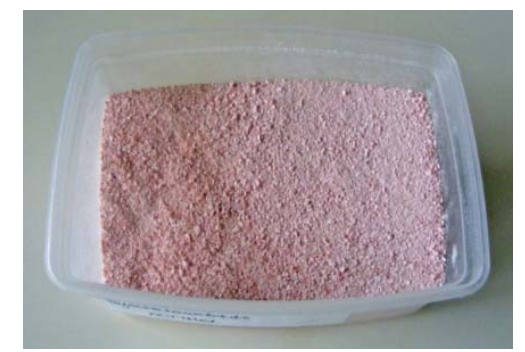

2/B picture. grinded selenium rich yoghurt powder

\section{Particle Size and Distribution Analysis}

In order to measure the size and distribution of the produced nano-particles we used Malvern Mastersizer 2000 particle size analyser. Which works by using the optical bench to capture the actual scattering pattern from a field of particles, then it calculates the size of particles that created the pattern.

\section{E. Method of Selenium Concentration Determination}

We used flame emission atomic absorption spectrometer (Thermo ICE 3000) and atomic fluorescence spectrometer (PSA Thermo, Excalibur) to determine the final selanium concentration of our nano selenium samples prepared. In sample preparation process in order to digest the samples we added $3 \mathrm{ml} \mathrm{cc} . \mathrm{HNO}_{3}$ and $5 \mathrm{ml} 30 \% \mathrm{H}_{2} \mathrm{O}_{2}$ to $1 \mathrm{ml}$ sample and heated at $120^{\circ} \mathrm{C}$ for 60 minutes. We filtered the digested samples and adjusted up to $50 \mathrm{ml}$ with purified water. In the purified selenium samples we measured 200-500 mg/l selenium concentration, while in the Lactomicrosel samples we measured $1000-3500 \mathrm{mg} / \mathrm{kg}$ selenium concentration.

\section{RESULtS}

\section{A. Particle Size and Distribution}

We measured the particle size and distribution of the purified selenium spheres produced by bacteria (particle size depends on the strain, this figure we show Lactobacillus casei), that are usually between $50-500 \mathrm{~nm}$. We realized that the distribution varies with the $\mathrm{pH}$, because the cohesion of the spheres depends on the $\mathrm{pH}$ of the liquid medium. At $\mathrm{pH}$ up to 10 particles form plaques, stick to each other (Figure 1.), but $\mathrm{pH}$ above 10 (Figure 2.), disintegrates the coherence of the particles.

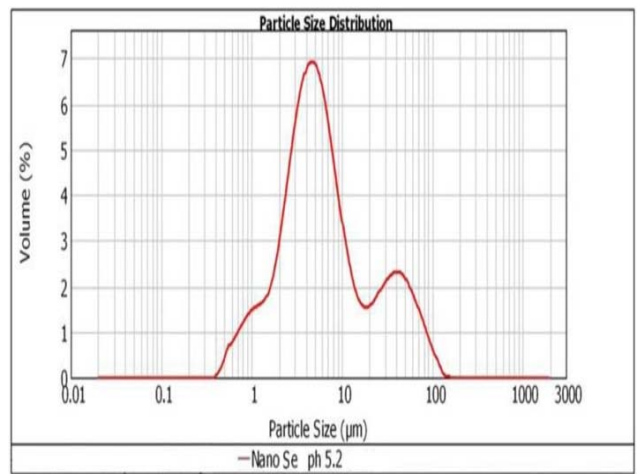

Figure 1.

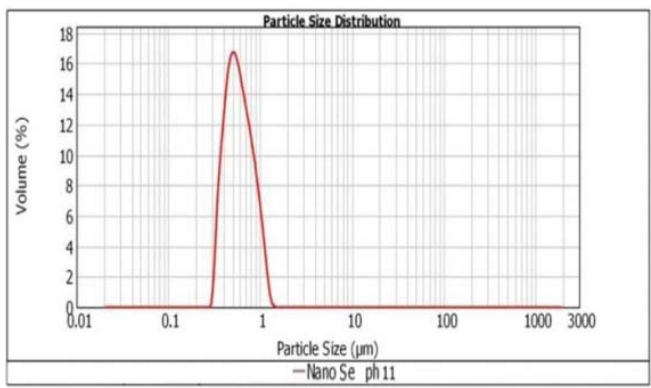

Figure 2.

\section{B. Elemental Selenium Transformation to Selenite}

We supposed that consumed elemental Nano-Se in a organic system continually transforms to selenite $\left(\mathrm{SeO}_{3}{ }^{2}-\right)$, maintaining the needed blood selenium concentration. In order to to prove this mechanism we centrifuged $50 \mathrm{ml}$ of Nano-Se suspension and re-suspended it in distillated water and measured the selenite concentration change in the supernatant for a few days. (Figure 3.)

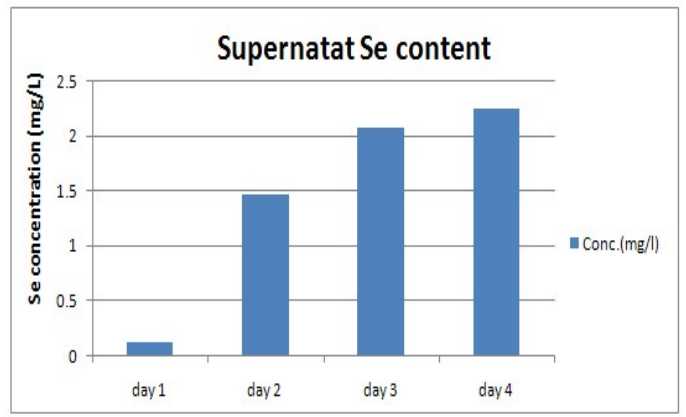

Figure 3.

\section{Scanning Electron Microscopic Pictures}

Purified elemental selenium nano-spheres under the microscope show very clear picture (Figure 4.).

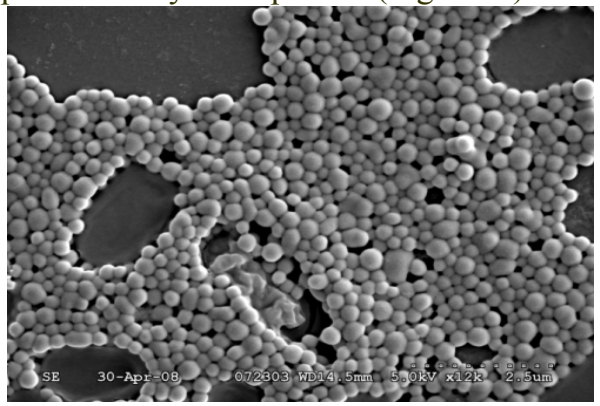

Figure 4. Electron microscopic picture of $250 \mathrm{~nm}$ sized selenium nano spheres 


\section{DISCUSSION}

Simplicity and rapidity of the technology and safety of the employed strains makes significant progress over the art in producing selenium nanospheres. Homogeneity and quality of the shape and size of elemental selenium nanoparticles produced chemically by other technology used in the art does not come close to the characteristics of the material prepared by the fermentation technology of the technology using e.g. lactic acid bacteria. The process of the technology is suitable for producing elemental selenium nanospheres sized $100-500 \mathrm{~nm}$, wherein the size distribution of nanospheres is generally characterized by a percentage deviation from the mean size of $5-20 \%$. Advantageously, the microorganism used in the process of the technology may be selected from the group consisting of the following species: Lactobacillus bulgaricus, Lactobacillus acidophillus, Bifidobacterium bifidum, Streptococcus thermophilus, Lactobacillus casei, Lactobacillus rhamnosus and Bifidobacterium longum.

Advantageously, when used as a food additive, the nanospheres produced according to the processes of the technology need not be fully purified or purified at all because the medium and the bacteria applied in the production process may be fully suitable for human or animal consumption. The technology further concerns isolated grey elemental selenium nanospheres directly obtainable by a process according to the technology. The technology also relates to the use of elemental selenium nanospheres obtainable by a process according to the technology in the food, microelectronic or optical industry.

\section{Conclusions}

A novel technology for producing selenium nanospheres in homogeneous in form and size within a short period of time (12-48 hours) has been developed. Elements with metallic properties have not been previously produced by fermentation using non-toxic aerobic bacteria, advantageously using bacteria permitted for use in the food industry. Selenium produced this way is exceptionally good raw material for forming nanosurfaces because of its homogeneous particle size distribution and regular, spherical shape. The technology developed is a manufacturing process which enables forming of a suspension as well as a powder containing valuable selenium spheres having unique characteristics. Material prepared in such a way can be used in the food industry as food or feed additive, in microelectronics as special semiconductor as well as in optical applications. The relative simplicity of the technology developed allows for significant decline in prices which can further broaden the range of useful high quality raw materials available.

\section{ACKNOWLEDGEMENT}

The authors wish to thank. Dr. Lajos Daróczi at the Department of Solid State Physics, University of Debrecen for the scanning electron microscopic pictures and Kinga Nagy for the help in the atomic absorption spectrometer measurements, Nanofood Lab., University of Debrecen.

\section{REFERENCES}

[1] Official Journal of the European Communities, Directive 2002/46/EC of the European parliament and of the council of 10 June 2002

[2] Cooke, T. D. \& Bruland, K. W. (1987). Aquatic Chemistry of Selenium - Evidence of Biomethylation. Environmental Science \& Technology, 21(12), 1214-1219.

[3] Emmie Dumont, Frank Vanhaecke, and Rita Cornelis (2006) Selenium speciation from food source to metabolites: a critical review. Anal Bioanal Chem.; 385(7): 1304-1323.

[4] Zayed AM, Lytle CM, Terry N (1998) Accumulation and volatilization of different chemical species of selenium by plants. Planta 206: 284-292

[5] Demirci, A., A. L. Pometto, III and J. D. Cox. (1999). Enhanced organically bound selenium yeast production by fed-batch fermentation. J. Agric. Food Chem. 47:2496-2500.

[6] Nair B. and Pradeep T. (2002) Coalescence of nanoclusters and the formation of sub-micron crystallites assisted by Lactobacillus strains. Cryst. Growth Des., 2: 293

[7] Schuler D. and Frankel R. B. (1999) Bacterial magnetosomes: microbiology, biomineralization and biotechnological applications. Appl. Microbiol. Biotechnol.,52: 464

[8] Moon J. W., Roh Y., Lauf R. J., Vali H., Yeary L. W., Phelps T. J. (2007) Microbial preparation of metal-substituted magnetite nanoparticles. Journal of microbiological methods 70(1), 150-58.

[9] Sastry, M., Ahmad, A., Islam, N.I., Kumar, R. (2003). Biosynthesis of metal nanoparticles using fungi and actinomycete. Current Sci. 85, 162.

[10] Gardea-Torresdey, J. G, Parsons, Gomez E., Peralta-Videa J., Troiani H. E., Santiago P. and Yacaman M. J. (2002) Formation and growth of au nanoparticles inside live alfalfa plants, Nanoletters., 2: 397

[11] Zhang J., Wang H., Yan X., Zhang L.(2004) Comparison of shortterm toxicity between Nano-Se and selenite in mice. Life Sciences 76(10); 1099-1109

[12] Xueyun Gao, Tao Gao and Lide Zhang J. Mater (2003). Solutionsolid growth of $\alpha$-monoclinic selenium nanowires at room temperature. Chem., 13, 6-8

[13] Wang H., Zhang J., Yu H. (2007) Elemental selenium at nano size possesses lower toxicity without compromising the fundamental effect on selenoenzymes: Comparison with selenomethionine in mice. Free Radical Biology \& Medicine 42, 1524-33.

[14] Oremland R. S., Herbel M. J., Blum J. S., Langley S., Beveridge T. J., Ajayan P. M., Sutto T., Ellis A. V., Curran S. (2004) Structural and spectral features of selenium nanospheres produced by Se-respiring bacteria. Applied and environmental microbiology 70(1), 52-60

[15] Fesharaki P. J,, Nazari P., Shakibaie M., Rezaie S., Banoee M., Abdollahi M. et al . (2010) Biosynthesis of selenium nanoparticles using Klebsiella pneumoniae and their recovery by a simple sterilization process. Braz. J. Microbiol. 41(2): 461-466.

[16] Prokisch, József (Debrecen, HU), Zommara, Mohsen A. (Alexandria, EG) 2010 Process for Producing Elemental Selenium Nanospheres United States 20100189634

Péter Eszenyi, biography and photograph not available at the time of publishing.

Attila Sztrik, biography and photograph not available at the time of publishing.

Beáta Babka, biography and photograph not available at the time of publishing.

József Prokisch, biography and photograph not available at the time of publishing. 